\title{
Besoins en soins de soutien des femmes atteintes de cancer du poumon
}

par Margaret I. Fitch et Rose Steele

\section{Abrégé}

Le principal objectif de cette étude exploratoire était de cerner les besoins en soins de soutien des femmes atteintes de cancer du poumon qui fréquentent un centre régional de cancérologie ambulatoire. L'incidence du cancer du poumon dépasse le domaine physique chez les personnes diagnostiquées de cette maladie et pourtant, relativement peu d'écrits portent sur leurs besoins en matière de soins de soutien, mis à part ceux qui concernent la prise en charge des symptômes.

Trente-quatre femmes diagnostiquées d'un cancer du poumon ont participé à cette étude exploratoire en remplissant un questionnaire d'autoévaluation (Enquête sur les besoins en matière de soins de soutien). Les données ainsi obtenues montraient clairement que ce groupe de femmes manifeste un large éventail de besoins et que bon nombre d'entre eux demeurent insatisfaits. Les besoins le plus fréquemment mentionnés étaient le manque d'énergie, la douleur et l'inquiétude concernant les proches. Les patientes relataient les difficultés qu'elles rencontraient sur le plan de la gestion de leurs besoins et beaucoup éprouvaient de la détresse émotionnelle du fait de ces difficultés. Des suggestions relatives à la pratique et aux futures études de recherche sont présentées afin d'aider les infirmières en oncologie à dispenser des soins optimaux à ce groupe de patientes.

La santé des femmes ou les perturbations subies par cette dernière constituent une question qui a reçu, ces dernières années, une attention accrue dans la littérature. On possède désormais un corpus de connaissances appuyant l'idée selon laquelle il existe des différences en matière de processus morbides qui peuvent dépendre $\mathrm{du}$ sexe (différences biologiques) ou du genre (différences socioculturelles). Il pourrait également être nécessaire de comprendre les différences relatives à l'adaptation et à la qualité de vie.

Le cancer du poumon est une des maladies où des différences ont été déterminées entre les hommes et les femmes. Ainsi, des différences ont été cernées en ce qui concerne la susceptibilité à la maladie (Siegfried, 2001), le type de cancer (Groff, 2002), le stade (Erridge, 2003) et la survie (Radzikowska, Glaz et Roszkowski, 2002). À l'heure actuelle, le cancer du poumon est la première cause de décès chez les Canadiennes. Il provoque autant de décès que le cancer du sein et l'ensemble des cancers gynécologiques réunis (Institut national du cancer du Canada [INCC], 2006). En 2006, 10600 femmes ont été diagnostiquées avec cette maladie et 8600 femmes en sont mortes. Les femmes préménopausées présentent plus souvent des cas de cancer avancé et subissent une résection chirurgicale plus étendue par rapport aux femmes plus âgées

Margaret I. Fitch, inf., PhD, Chef, Soins infirmiers en oncologie et Soins de soutien, Centre régional de cancérologie de TorontoSunnybrook, 2075 Bayview Ave., Toronto, ON M4N 3 M5

Courriel : marg.fitch@sunnybrook.ca

Rose Steele, inf., PhD, Professeure agrégée, École des sciences infirmières, Université York, Toronto, ON (postménopausées), mais possèdent un avantage sur le plan de la survie après l'ajustement des covariables (Moore, Mery, Jaklitsch, Estocain, Bueno, Swanson et coll., 2003). En outre, les femmes postménopausées présentent un avantage en matière de survie par rapport aux hommes du même âge (Moore et coll., 2003).

Malgré les récentes avancées sur le plan du traitement, le cancer du poumon demeure encore, dans la majorité des cas, une maladie incurable. Chez les femmes, la survie relative à 5 ans s'élève à $18 \%$ (INCC, 2006). Le diagnostic de cancer du poumon et son traitement ultérieur ont une incidence physique sur les personnes touchées ainsi que des répercussions sociales, psychologiques et émotionnelles. De graves perturbations sur le plan de la qualité de vie, particulièrement les aspects sociaux et psychologiques, ont été signalées davantage chez les plus jeunes femmes présentant un plus fort risque (Sarna, Brown, Cooley, Williams, Chernecky, Padilla et coll., 2005). Les perturbations de la qualité de vie sont souvent reliées à la sévérité des symptômes et des déficiences physiques (Hopwood et Stephens, 2000).

Les infirmières jouent un rôle clé en évaluant les réponses des patientes face à la maladie et en les aidant à gérer les défis relatifs à la détresse liée aux symptômes ou aux déficiences physiques croissantes. Jusqu'à présent, le corpus de connaissances qui permettrait de guider la pratique infirmière relativement aux femmes atteintes de cancer du poumon reste peu développé. Alors qu'on dispose de certaines informations sur les besoins en matière de soins de soutien des personnes atteintes de cancer, il existe peu de connaissances sur le type d'aide qui conviendrait aux femmes atteintes de cancer du poumon au long de leur expérience de la maladie.

\section{Recension des écrits}

Étant donné sa fréquence relative et la faiblesse des taux de survie (INCC , 2006), le cancer du poumon est la première cause de décès prématuré dû au cancer. Par conséquent, chez la plupart des patients atteints de cancer du poumon, la maladie évolue rapidement vers une issue fatale. À mesure que les symptômes apparaissent et gagnent en sévérité, les patients atteints de cancer du poumon éprouvent souvent de la difficulté à gérer leurs activités journalières. La documentation scientifique fait état de symptômes tels que la dyspnée (p. ex. Edmonds, Karlsen, Khan et Addington-Hall, 2001; Krishnasamy, Wilkie et Haviland, 2001; Sarna et coll., 2004); la toux (p. ex. Bailey, Parmar et Stephens, 1998; Given, Given et Stommel, 1994; Hopwood et Stephens, 1995; Sarna et coll., 2004); l'anorexie (p. ex. Sarna, Lindsey, Dean, Brecht et McCorkle, 1994) et la perte pondérale (p. ex. Brown et Radke, 1998). Dans une récente étude visant à mieux comprendre l'expérience des symptômes chez les personnes atteintes de cancer du poumon, la fatigue et la douleur étaient les symptômes le plus souvent mentionnés (Kiteley et Fitch, 2006). De plus, les participants à l'étude ci-dessus indiquaient qu'ils devaient composer simultanément avec plusieurs symptômes. Malheureusement, la plus grande partie des écrits publiés ne rapporte pas les données sous forme d'observations sexospécifiques.

Des liens ont été récemment établis entre la sévérité des symptômes physiques et des limitations fonctionnelles, d'une part, et la qualité de vie des patients atteints de cancer du poumon, d'autre part. Hopwood et Stephens (2000) ont fait état de la présence courante et persistante de la dépression chez les patients ayant le cancer du poumon et de l'importance de l'incapacité fonctionnelle à titre de facteur de risque. La dépression croissait de $41 \%$ avec chaque 
incrément d'incapacité fonctionnelle. Trippoli, Varani, Lucioni et Messori (2001) ont avancé que c'est le cancer métastatique, avec l'augmentation de la douleur physique et la diminution du fonctionnement physique, qui exerce la plus grande incidence sur la qualité de vie. Des fluctuations concernant la qualité de vie ont également été signalées chez les patients atteints de cancer du poumon tandis qu'ils suivent leur traitement (John, 2001; Hollen, Gralla et Rittenberg, 2004).

Il en ressort que le diagnostic de cancer et le traitement ont un impact qui dépasse le domaine physique. Des répercussions sociales, émotionnelles, psychologiques, spirituelles et pratiques font fréquemment leur apparition chez les personnes ayant le cancer (Ashbury, Findley, Reynolds et McKerracher, 1998; Charles, Sellick, Montesanto et Mohide, 1996; Whelan et coll., 1997). En particulier, l'évolution de la maladie et des symptômes et leur aggravation peuvent amener les patients à rencontrer tout un éventail de difficultés et de défis dans leurs efforts d'adaptation à leur circonstances (Bradley, Davis et Chow, 2005; Sarna et coll., 2004; Bruera et coll., 2001).

La détresse psychosociale est une expérience fréquente chez les patients atteints de cancer, notamment durant la période qui suit le diagnostic et les périodes marquées par la détérioration de leur état. Zabora, Brintzenhofeszoc, Curbow, Hooker et Piantadosi (2001) ont constaté qu'environ $20 \%$ des patients atteints de cancer éprouvent un niveau de détresse beaucoup plus élevé après le diagnostic. Krishnasamy et ses collègues (2001) ont signalé qu'environ les deux tiers des patients atteints de cancer du poumon de leur étude décrivaient des sentiments d'anxiété tandis que les prestataires de soins estimaient que l'anxiété constituait un problème chez moins d'un quart des patients. Morasso et ses collègues (1999) déclaraient qu'il existait un rapport significatif entre un fonctionnement moindre et des besoins insatisfaits dans les domaines des soins personnels, de l'information, de la communication, du fonctionnement professionnel et de la proximité affective. De plus, les patients dont les besoins ne sont pas satisfaits éprouvent un degré significativement plus élevé de détresse psychologique et de détresse liée aux symptômes. Dans une étude à méthode mixte, Maliski, Sarna, Evangelista et Padilla (2003) ont découvert que $31 \%$ des survivants du cancer du poumon étaient déprimés et qu'ils étaient plus susceptibles d'entretenir une conception plus négative de la vie que le groupe des non-déprimés. Bradley et ses collègues (2005) ont indiqué que $61 \%$ des patients fréquentant leur clinique $(\mathrm{n}=1070)$ souffraient de dépression. La dépression variait de modérée à sévère chez $30 \%$ d'entre eux. Ces auteurs ont suggéré que la qualité de vie des patients pourrait être rehaussée si les professionnels de la santé fournissaient des interventions adéquates.

Fallen et Schmit (2004) ont rapporté que la dépression comme mode d'adaptation au cancer du poumon était associée à une survie de moindre durée tandis que Sarna et ses collaborateurs (2005) constataient que $35 \%$ des 217 femmes atteintes de cancer du poumon composant leur échantillon étaient déprimées et que $36 \%$ d'entre elles entretenaient des conceptualisations négatives de la maladie. Soixante-quinze pour cent des membres de cet échantillon signalaient que la détresse émotionnelle, la détresse familiale et l'impact de leur maladie et de ses séquelles sur le fonctionnement sexuel nuisaient à leur qualité de vie. Chapple, Ziebland et MacPherson (2004) ont décrit l'effet profond qu'exercent les stigmates du cancer du poumon sur les personnes qui en sont atteintes. De plus, Sarna et ses collègues (2004) ont signalé que $71 \%$ des 142 survivants à 5 ans du cancer du poumon se déclaraient pleins d'espoir et $50 \%$ considéraient que l'expérience du cancer s'accompagnait de changements positifs dans leur vie. Dans cette étude, les prédicteurs primaires d'une qualité de vie moindre étaient l'ethnicité et la dépression.

Faire face aux répercussions du cancer du poumon et de son traitement peut représenter un fardeau pour la personne atteinte et pour sa famille. Lorsqu'elles reçoivent une information appropriée et bénéficient de relations thérapeutiques adéquates avec l'équipe de soins en cancérologie, certaines personnes sont capables de mobiliser leurs propres ressources afin de s'adapter aux expériences liées au cancer; d'autres, au contraire, ont besoin d'une aide supplémentaire. Cependant, on sait très peu de choses sur les divers types d'aide exigés par ces personnes. Il convient de noter que Bruera et ses collègues (2001) ont démontré que les patients $(n=166)$ obtenaient une amélioration statistiquement significative de leur score psychosocial quand ils bénéficiaient de l'aide d'une équipe multidisciplinaire.

Bien que l'on dispose d'information sur les besoins en soins de soutien des patients atteints de cancer du poumon, on en sait peu sur l'éventail complet des besoins des femmes ayant un cancer du poumon. La création d'un tel corpus de connaissances est importante pour plusieurs raisons: 1) pouvoir planifier les soins individuels des patientes, 2) pouvoir prendre des décisions sur l'affectation des ressources, 3) dégager les domaines à privilégier pour améliorer la qualité des soins dispensés.

\section{But}

Le but premier de cette étude descriptive transversale était de cerner les besoins (physiques, émotionnels, sociaux, spirituels, psychologiques et pratiques) des femmes atteintes d'un cancer du poumon qui fréquentent un centre de cancérologie ambulatoire offrant une gamme complète de services. Le second but était d'explorer la détresse émotionnelle que les femmes percevaient du fait de la nonsatisfaction des besoins qu'elles éprouvaient.

\section{Méthodologie}

\section{Procédure}

L'étude a débuté lorsque ses auteures ont reçu l'approbation éthique de l'établissement en question et de l'université d'une des chercheuses. Une associée de recherche a consulté le personnel de la clinique des maladies du poumon afin d'identifier des participantes éventuelles. Les patientes fréquentant la clinique étaient admissibles à participer à condition qu'elles aient dix-huit ans ou plus, aient un diagnostic de cancer du poumon et ne manifestent aucun signe de métastases cérébrales ou de détresse émotionnelle excessive. Les patientes ont été abordées dans la zone d'accueil par l'associée de recherche qui leur a expliqué les objectifs et la méthodologie de l'étude. De l'information verbale a été donnée aux patientes pour quelles soient au courant de leurs droits relativement à la participation, puis l'associée de recherche a invité les patientes à participer. Celles qui ont accepté de le faire ont signé un formulaire de consentement en double. La patiente en gardait une copie tandis que l'associée de recherche conservait l'autre.

L'associée de recherche a remis à chaque participante un questionnaire d'autoévaluation (Enquête sur les besoins en matière de soins de soutien) afin qu'elle le remplisse et a déterminé si la patiente avaient besoin ou non d'aide pour le faire. Dans ce cas (p. ex. à cause d'une incapacité physique), l'associée de recherche accompagnait la patiente vers un lieu privé et tranquille et remplissait le questionnaire dans le cadre d'une entrevue. En ce qui concerne les patientes ayant accepté de remplir elles-mêmes le questionnaire durant leur visite à la clinique, l'associée de recherche retournait voir la patiente à une heure spécifiée à l'avance afin de recueillir son questionnaire. On a également placé, dans la clinique, une boîte dans laquelle les patientes pouvaient déposer leur questionnaire et ce, dans une enveloppe cachetée. Enfin, si des patientes n'avaient pas la possibilité de remplir le questionnaire durant leur visite à la clinique, l'associée de recherche leur remettait une enveloppe affranchie portant l'adresse requise pour l'envoi par la poste.

L'instrument de mesure (c.-à-d. l'enquête sur les besoins en matière de soins de soutien) était uniquement disponible en anglais. Toutefois, afin de minimiser l'exclusion des patientes qui ne parlaient ni ne lisaient l'anglais ou n'écrivaient pas dans cette langue, on 
invitait des proches ou des amis ayant une bonne maîtrise de cette langue d'expliquer l'étude aux patientes et de fournir des services d'interprétation si la patiente et eux-mêmes étaient d'accord. Toutes les réponses étaient inscrites en anglais sur le formulaire. La patiente signait le formulaire de consentement et l'interprète la signait également en y apposant une note qui indiquait que la patiente avait donné son libre consentement. L'associée de recherche a noté les diverses manières dont les questionnaires ont été remplis (par la patiente elle-même, dans le cadre d'une entrevue avec l'associée de recherche ou par l'intermédiaire d'un ou une interprète). Aucune différence n'a été remarquée entre ces groupes lors de l'analyse. L'associée de recherche a également consulté le dossier médical des patientes afin de compléter les renseignements démographiques et de connaître la date à laquelle des métastases avaient été diagnostiquées pour la première fois chez les patientes.

\section{Collecte et analyse des données}

Au départ, les patientes ont rempli une feuille de renseignements démographiques comprenant huit questions : âge (en années); situation maritale (mariée/en union civile; séparée/divorcée; veuve; célibataire); plus haut niveau de scolarité atteint (aucune scolarité officielle; école primaire; école secondaire; programme collégial; programme universitaire); type de cancer; mois et année du diagnostic initial de cancer; traitement reçu pour ce cancer (radiothérapie; chimiothérapie; chirurgie; autre traitement); et traitement actif actuel pour le cancer (radiothérapie; chimiothérapie; chirurgie; autre traitement). Pour les deux questions concernant le traitement, les patientes pouvaient cocher toutes les réponses qui s'appliquaient à leur cas.

Ensuite, les patientes ont rempli l'enquête sur les besoins en matière de soins de soutien adaptée à la population des personnes ayant le cancer du poumon. L'instrument qui a servi de base au questionnaire utilisé dans le cadre de l'étude était la Supportive Care Needs Survey dont la conception et la validation originales étaient survenues en Australie (Bonevski et coll., 2000). Cet instrument comprend 60 items à question fermée auxquels les patientes répondent au moyen d'une échelle à 5 points. Cette dernière reflète divers niveaux de besoin allant de $1=$ «Aucun besoin » (l'item n'était pas un problème pour la patiente du fait de son cancer) à $5=$ «Des besoins» c.-à-d. un grand besoin d'aide (l'item était d'une grande importance ou une grande préoccupation pour la patiente et cette dernière avait énormément besoin d'une aide supplémentaire pour composer avec le problème ou l'enjeu). L'instrument de mesure est facilement compris par les individus ayant suivi une scolarité minimale et il faut compter environ 20 minutes pour le remplir (Bonevski et coll., 2000).

Tableau 1. Variables démographiques :

Patientes atteintes de cancer du poumon $(n=34)$

\begin{tabular}{|l|l|l|}
\hline Variable & Résultat & Nombre \\
\hline Âge & $<50$ ans & 4 \\
& $50-70$ ans & 20 \\
& $<70$ ans & 10 \\
\hline Situation maritale & Mariée & 24 \\
& Séparée/divorcée/veuve & 10 \\
\hline Niveau de scolarité & $<$ Collège & 24 \\
& Collège & 10 \\
\hline Temps depuis & $<1$ an & 4 \\
le diagnostic & $1-2$ ans & 15 \\
& $2+$ ans & 15 \\
\hline
\end{tabular}

Une analyse factorielle en composantes principales (Bonevski et coll., 2000) a révélé que les besoins pouvaient être regroupés en fonction de cinq facteurs: 1) besoins psychologiques-besoins liés aux émotions et à l'adaptation; 2) système de santé et information sur la santé - besoins liés au centre de traitement et à l'information sur la maladie, le traitement et le suivi; 3) besoins physiques et activités quotidiennes-besoins liés à l'adaptation aux symptômes physiques, aux effets secondaires du traitement et à l'exécution des tâches et activités physiques habituelles; 4) soins et soutien de la patientebesoins liés aux fournisseurs de soins de santé qui doivent être sensibles aux besoins physiques et émotionnels, au respect de la vie privée et du libre choix; 5) sexualité-besoins liés aux rapports sexuels. Les coefficients alpha de Cronbach correspondant aux cinq facteurs allaient de 0,87 pour la sexualité à 0,97 pour le facteur psychologique. Quatre autres items n'étaient pas associés à une saturation factorielle particulière soit le transport, les attitudes d'autrui envers la patiente, les finances et pouvoir parler avec d'autres personnes.

L'instrument de mesure a subi des révisions afin de pouvoir être employé avec les patientes atteintes d'un cancer du poumon de la présente étude. Un essai pilote de la version originale de l'instrument de Bonevski auprès des patientes du centre a entraîné un réordonnancement de certaines des questions afin d'en rehausser la clarté. De légères modifications ont également été apportées au libellé des questions en vue de mieux refléter le milieu canadien. Par exemple, le terme «hôpital» a été remplacé par les mots «centre de cancérologie». L'enquête sur les besoins en matière de soins de soutien aborde les sept domaines des soins de soutien au moyen de 61 items: besoins émotionnels (11 items); besoins d'information (9 items); besoins physiques (symptômes; 11 items); besoins pratiques (7 items); besoins psychologiques (11 items); besoins sociaux (5 items); besoins spirituels (7 items). La fidélité des résultats obtenus auprès des patientes atteintes d'un cancer du poumon dans le cadre de l'étude était encourageante avec des sous-échelles (domaines) se situant entre 0,66 et 0,90,6 sur 7 étant égaux ou supérieurs à 0,80 . L'analyse effectuée pour l'étude était de nature descriptive et privilégiait la fréquence des items et les cotes des sous-échelles.

\section{Échantillon}

Un échantillon de convenance, composé de 34 femmes, a participé à l'étude en remplissant l'enquête sur les besoins en matière de soins de soutien. Il représente approximativement le tiers des femmes suivies par la clinique du centre en un mois. Similaire en cela à la population de la clinique, les femmes de l'échantillon étaient d'un âge plus avancé, plus de $80 \%$ d'entre elles ayant plus de 60 ans. La plupart des patientes étaient mariées $(n=24)$, plus d'un tiers d'entre elles avaient obtenu, au minimum, une éducation collégiale $(n=10)$ et $44 \%$ avaient été diagnostiquées entre 12 et 24 mois auparavant (voir le tableau 1). Environ $92 \%$ des patientes rapportaient qu'elles suivaient un traitement. Ce dernier incluait la chimiothérapie, la radiothérapie, la chirurgie ou une combinaison de ces modalités.

\section{Résultats}

\section{Les problèmes actuels}

Dans leurs réponses à l'enquête, les patientes ont signalé les problèmes auxquels elles faisaient face à ce moment-là. Les femmes de l'étude rapportaient être aux prises avec des problèmes dans les sept domaines. Quasiment tous les items ont été mentionnés par au moins une répondante. Le nombre de patientes faisant état d'un problème s'étendait de $n=1$ (besoins sociaux - «que les parents et amis aient le droit d'être avec vous à l'hôpital quand vous le désirez») à $n=28$ (besoins psychologiques - «peur que le cancer se propage»). Tous les domaines, à l'exception de celui de l'information, avaient au moins un item pour lequel un tiers ou plus des femmes rapportaient faire face à un problème. 


\section{Besoins physiques}

Six items constituaient des problèmes pour plus d'un tiers des participantes : manque d'énergie $(n=23)$; ne plus être capable de faire des choses qu'on pouvait faire auparavant $(n=22)$; essoufflement $(n=17)$; ne plus être capable de faire les travaux ménagers $(n=17)$; toux $(n=14)$; se sentir mal $(n=14)$ (voir le tableau 2$)$.

\section{Besoins émotionnels}

Plus d'un tiers des femmes signalaient éprouver des difficultés relativement à cinq items : se sentir déprimé $(n=18)$; sentiments de tristesse $(n=16)$; anxiété $(n=16)$; s'ennuyer ou se sentir inutile $(n=14)$; et s'inquiéter de n'exercer aucun contrôle sur les résultats du traitement $(\mathrm{n}=13)$ (voir le tableau 3).

\section{Tableau 2. Items du domaine physique $(\mathrm{N}=34)$}

\begin{tabular}{|c|c|c|c|c|c|c|c|c|}
\hline \multirow{3}{*}{ Item } & \multirow{2}{*}{\multicolumn{2}{|c|}{$\begin{array}{l}\text { Femmes sans } \\
\text { inquiétudes }\end{array}$}} & \multicolumn{6}{|c|}{ Femmes ayant des inquiétudes } \\
\hline & & & \multicolumn{2}{|c|}{ Détresse minime } & \multicolumn{2}{|c|}{ Détresse modérée } & \multicolumn{2}{|c|}{ Détresse élevée } \\
\hline & Nombre & $\%$ & Nombre & $\%$ & Nombre & $\%$ & Nombre & $\%$ \\
\hline Manque d'énergie & 11 & 32,4 & 10 & 29,4 & 7 & 20,6 & 6 & 17,6 \\
\hline $\begin{array}{l}\text { Ne pas être capable de faire les choses } \\
\text { que vous faisiez }\end{array}$ & 12 & 35,3 & 9 & 26,5 & 11 & 32,4 & 2 & 5,9 \\
\hline Essoufflement & 17 & 50,0 & 10 & 29,4 & 7 & 20,6 & - & - \\
\hline Ne pas être capable de faire les travaux ménagers & 17 & 50,0 & 3 & 8,8 & 12 & 35,3 & 2 & 5,9 \\
\hline Se sentir mal & 20 & 58,8 & 10 & 29,4 & 3 & 8,8 & 1 & 2,9 \\
\hline Toux & 20 & 58,8 & 7 & 20,6 & 6 & 17,6 & 1 & 2,9 \\
\hline Douleur & 23 & 67,6 & 8 & 23,5 & 3 & 8,8 & - & - \\
\hline $\begin{array}{l}\text { Changements touchant le } \\
\text { fonctionnement sexuel }\end{array}$ & 23 & 67,6 & 3 & 8,8 & 6 & 17,6 & 2 & 5,9 \\
\hline Perte d'appétit & 24 & 70,6 & 7 & 20,6 & 1 & 2,9 & 2 & 5,9 \\
\hline Nausée/vomissements & 31 & 91,2 & 2 & 5,9 & - & - & 1 & 2,9 \\
\hline $\begin{array}{l}\text { Le personnel du centre répond } \\
\text { promptement aux besoins }\end{array}$ & 31 & 91,2 & 1 & 2,9 & 1 & 2,9 & 1 & 2,9 \\
\hline
\end{tabular}

Tableau 3. Items du domaine émotionnel $(\mathrm{N}=34)$

\begin{tabular}{|c|c|c|c|c|c|c|c|c|}
\hline \multirow{3}{*}{ Item } & \multirow{2}{*}{\multicolumn{2}{|c|}{$\begin{array}{l}\text { Femmes sans } \\
\text { inquiétudes }\end{array}$}} & \multicolumn{6}{|c|}{ Femmes ayant des inquiétudes } \\
\hline & & & \multicolumn{2}{|c|}{ Détresse minime } & \multicolumn{2}{|c|}{ Détresse modérée } & \multicolumn{2}{|c|}{ Détresse élevée } \\
\hline & Nombre & $\%$ & Nombre & $\%$ & Nombre & $\%$ & Nombre & $\%$ \\
\hline Se sentir déprimée & 16 & 47,1 & 8 & 23,5 & 7 & 20,6 & 3 & 8,8 \\
\hline Anxiété & 18 & 52,9 & 7 & 20,6 & 7 & 20,6 & 2 & 5,9 \\
\hline Sentiments de tristesse & 18 & 52,9 & 7 & 20,6 & 7 & 20,6 & 2 & 5,9 \\
\hline S'ennuyer ou se sentir inutile & 20 & 58,8 & 6 & 17,6 & 5 & 14,7 & 3 & 8,8 \\
\hline $\begin{array}{l}\text { S'inquiéter de n'exercer aucun contrôle } \\
\text { sur les résultats du traitement }\end{array}$ & 21 & 61,8 & 5 & 14,7 & 5 & 14,7 & 3 & 8,8 \\
\hline $\begin{array}{l}\text { Anxiété à l'idée de subir un } \\
\text { traitement quelconque }\end{array}$ & 23 & 67,6 & 6 & 17,6 & 3 & 8,8 & 2 & 5,9 \\
\hline Changements sur le plan des rapports sexuels & 25 & 73,5 & 3 & 8,8 & 5 & 14,7 & 1 & 2,9 \\
\hline $\begin{array}{l}\text { Avoir un membre du personnel du centre avec } \\
\text { qui vous pouvez parler }\end{array}$ & 29 & 85,3 & 4 & 11,8 & 1 & 2,9 & - & - \\
\hline $\begin{array}{l}\text { La possibilité de parler à quelqu'un qui } \\
\text { comprenne et a vécu une expérience similaire }\end{array}$ & 29 & 85,3 & 1 & 2,9 & 2 & 5,9 & 2 & 5,9 \\
\hline $\begin{array}{l}\text { Être traitée en tant que personne et non } \\
\text { en tant que cas }\end{array}$ & 31 & 91,2 & 3 & 8,8 & - & - & - & - \\
\hline $\begin{array}{l}\text { Le personnel du centre reconnaît vos } \\
\text { sentiments et se montre sensible envers eux }\end{array}$ & 31 & 91,2 & 1 & 2,9 & 2 & 5,9 & - & - \\
\hline
\end{tabular}




\section{Besoins psychologiques}

Les six items pour lesquels plus d'un tiers des femmes ont rapporté éprouver actuellement un problème étaient les suivants : peur que le cancer se propage $(n=28)$; peur que le cancer revienne $(n=25)$; peur liée à la douleur $(\mathrm{n}=18)$; peur liée à l'incapacité or à la détérioration physique $(n=14)$; peur de perdre son indépendance $(n=13)$; apprendre à se sentir maître de la situation $(n=12)$ (voir le tableau 4).

\section{Besoins spirituels}

Un seul des items relatifs au domaine de la spiritualité a été signalé comme étant un problème actuel par plus d'un tiers des patientes : éprouver de l'incertitude au sujet de l'avenir $(n=19)$ (voir le tableau $5)$.

\section{Besoins sociaux}

«Vous vous souciez des inquiétudes de vos proches à votre égard» $(n=19)$ était le seul item que plus d'un tiers des femmes ont classé parmi les problèmes actuels (voir le tableau 6).

\section{Besoins d'information}

Aucun des items du domaine de l'information ne représentait un problème pour plus d'un tiers des femmes atteintes de cancer du poumon. L'item le plus fréquemment cité comme problème était «être informée des choses que je peux faire» $(n=9)$ (Voir le tableau 7).

\section{Besoins pratiques tttttttttttttttt}

Items le plus fréquemment mentionnés et détresse autodéclarée

Les dix problèmes mentionnés le plus fréquemment (par plus de $50 \%$ des patientes), relevaient du domaine physique (manque d'énergie, ne plus être capable de faire ce que vous faisiez auparavant, ne plus être capable de faire les travaux ménagers, essoufflement), du domaine psychologique (peur que le cancer se propage, peur que le cancer revienne et peur relative à la douleur), du domaine social (Vous vous souciez des inquiétudes de vos proches à votre égard), du

Tableau 4. Items du domaine social $(\mathrm{N}=34)$

\begin{tabular}{|c|c|c|c|c|c|c|c|c|}
\hline \multirow{3}{*}{ Item } & \multirow{2}{*}{\multicolumn{2}{|c|}{$\begin{array}{l}\text { Femmes sans } \\
\text { inquiétudes }\end{array}$}} & \multicolumn{6}{|c|}{ Femmes ayant des inquiétudes } \\
\hline & & & \multicolumn{2}{|c|}{ Détresse minime } & \multicolumn{2}{|c|}{ Détresse modérée } & \multicolumn{2}{|c|}{ Détresse élevée } \\
\hline & Nombre & $\%$ & Nombre & $\%$ & Nombre & $\%$ & Nombre & $\%$ \\
\hline Inquiétudes et soucis à propos de vos proches & 15 & 44,1 & 7 & 20,6 & 5 & 14,7 & 7 & 20,6 \\
\hline $\begin{array}{l}\text { Inquiétudes à propos de ceux de vos proches } \\
\text { qui prennent soin de vous }\end{array}$ & 23 & 67,8 & 3 & 8,8 & 4 & 11,8 & 4 & 11,8 \\
\hline $\begin{array}{l}\text { Changements dans les attitudes et } \\
\text { comportements d'autrui }\end{array}$ & 28 & 82,4 & 2 & 5,9 & 3 & 8,8 & 1 & 2,9 \\
\hline Parler du cancer avec d'autres personnes & 30 & 88,2 & 1 & 2,9 & 3 & 8,8 & - & - \\
\hline $\begin{array}{l}\text { Que des parents et amis aient le droit } \\
\text { d'être avec vous... }\end{array}$ & 33 & 97,1 & 1 & 2,9 & - & - & - & - \\
\hline
\end{tabular}

Tableau 5. Items du domaine psychologique $(\mathrm{N}=34)$

\begin{tabular}{|c|c|c|c|c|c|c|c|c|}
\hline \multirow{3}{*}{ Item } & \multirow{2}{*}{\multicolumn{2}{|c|}{$\begin{array}{l}\text { Femmes sans } \\
\text { inquiétudes }\end{array}$}} & \multicolumn{6}{|c|}{ Femmes ayant des inquiétudes } \\
\hline & & & \multicolumn{2}{|c|}{ Détresse minime } & \multicolumn{2}{|c|}{ Détresse modérée } & \multicolumn{2}{|c|}{ Détresse élevée } \\
\hline & Nombre & $\%$ & Nombre & $\%$ & Nombre & $\%$ & Nombre & $\%$ \\
\hline Peur que le cancer se propage & 6 & 17,6 & 12 & 35,3 & 9 & 26,5 & 7 & 20,6 \\
\hline Peur que le cancer revienne & 9 & 26,5 & 9 & 26,5 & 9 & 26,5 & 7 & 20,6 \\
\hline Peur à propos de la douleur & 16 & 47,1 & 8 & 23,5 & 6 & 17,6 & 4 & 11,8 \\
\hline Craintes relatives à l'incapacité physique & 20 & 58,8 & 6 & 17,6 & 4 & 11,8 & 4 & 11,8 \\
\hline Craintes relatives à la perte d'autonomie & 21 & 61,8 & 6 & 17,6 & 2 & 5,9 & 5 & 14,7 \\
\hline Apprendre à se sentir maître de la situation & 22 & 64,7 & 5 & 14,7 & 6 & 17,6 & 1 & 2,9 \\
\hline Accepter les changements liés à l'apparence & 25 & 73,5 & 5 & 14,7 & 2 & 5,9 & 2 & 5,9 \\
\hline Être rassurée par le personnel médical que... & 29 & 85,3 & 3 & 8,8 & 2 & 5,9 & - & - \\
\hline Avoir accès à un counseling professionnel & 31 & 91,2 & 2 & 5,9 & 1 & 2,9 & - & - \\
\hline $\begin{array}{l}\text { Être traitée dans un hôpital ou une } \\
\text { clinique de cancérologie }\end{array}$ & 32 & 94,1 & 2 & 5,9 & - & - & - & - \\
\hline $\begin{array}{l}\text { Meilleure protection du droit au respect } \\
\text { de la vie privée }\end{array}$ & 32 & 94,1 & - & - & 2 & 5,9 & - & - \\
\hline
\end{tabular}


domaine spirituel (incertitude à propos de l'avenir) et enfin, du domaine émotionnel (se sentir déprimé) (voir le tableau 9).

Les femmes qui déclaraient éprouver un problème étaient invitées à indiquer l'ampleur de la détresse qu'elles ressentaient du fait de ce problème particulier. Les tableaux 2-9 illustrent l'ampleur de la détresse signalée pour chacun des items dont l'intensité pouvait être faible, modérée ou forte. Il convient de noter que pour 9 des 10 items le plus fréquemment mentionnés, des sentiments de détresse d'intensité modérée à forte étaient rapportés par plus de $50 \%$ des participantes déclarant avoir de la difficulté avec l'item.

\section{Discussion}

Cette étude exploratoire a été entreprise en vue de décrire l'éventail complet des besoins en matière de soins de soutien des femmes atteintes d'un cancer pulmonaire. Étant donnée la méthodologie retenue pour l'étude, les données fournissent un instantané du fardeau potentiel, sur le plan des besoins, sous lequel croule cette population de patientes. Il est évident que les femmes éprouvaient des difficultés qui dépassaient la sphère physique et qu'elles faisaient face, simultanément, à toute une gamme de problèmes. En outre, chez une proportion assez importante d'entre elles, les problèmes qu'elles tentaient de résoudre provoquaient une forte détresse.

Une grande partie des items individuels liés à la sphère physique ont été signalés dans d'autres articles : le manque d'énergie ou la fatigue (Bradley et coll., 2005), la douleur (Bruera et coll., 2001), la dyspnée (O'Driscoll, Corner et Bailey, 1999) et enfin, l'incapacité de réaliser les activités quotidiennes ou de faire ce que l'on voudrait faire (Kenneth et Payne, 2005). Voogt et ses collègues (2005) ont établi un lien entre le manque d'émotions positives chez 105 patientes atteintes de cancer et leur incapacité de participer à des activités chargées de sens et la réduction du fonctionnement cognitif et social ainsi que l'accroissement de la fatigue. Une fatigue accablante peut amoindrir la capacité de la personne à exécuter les activités normales et quotidiennes, et les pertes résultantes peuvent avoir un effet dévastateur sur le bien-être de la personne (Kennett et Payne, 2005). De plus, il existe des résultats de recherche indiquant comment il convient de prendre en charge la fatigue et la dyspnée chez les

Tableau 6. Items du domaine de l'information ( $N=34)$

\begin{tabular}{|c|c|c|c|c|c|c|c|c|}
\hline \multirow{3}{*}{ Item } & \multirow{2}{*}{\multicolumn{2}{|c|}{$\begin{array}{l}\text { Femmes sans } \\
\text { inquiétudes }\end{array}$}} & \multicolumn{6}{|c|}{ Femmes ayant des inquiétudes } \\
\hline & & & \multicolumn{2}{|c|}{ Détresse minime } & \multicolumn{2}{|c|}{ Détresse modérée } & \multicolumn{2}{|c|}{ Détresse élevée } \\
\hline & Nombre & $\%$ & Nombre & $\%$ & Nombre & $\%$ & Nombre & $\%$ \\
\hline Être informée des choses que vous pouvez faire & 25 & 73,5 & 4 & 11,8 & 4 & 11,8 & 1 & 2,9 \\
\hline $\begin{array}{l}\text { Recevoir de l'information écrite sur les aspects } \\
\text { importants de vos soins }\end{array}$ & 26 & 76,5 & 5 & 14,7 & 3 & 8,8 & - & - \\
\hline $\begin{array}{l}\text { Recevoir de l'information sur divers } \\
\text { aspects de la gestion de vos soins }\end{array}$ & 28 & 82,4 & 1 & 2,9 & 5 & 14,7 & - & - \\
\hline $\begin{array}{l}\text { Etre informée le plus tôt possible des } \\
\text { résultats des analyses de laboratoire }\end{array}$ & 28 & 82,4 & 3 & 8,8 & 3 & 8,8 & - & - \\
\hline Être informée des groupes de soutien & 29 & 85,3 & 2 & 5,9 & 2 & 5,9 & 1 & 2,9 \\
\hline Obtenir des explications sur les analyses & 29 & 85,3 & 3 & 8,8 & 2 & 5,9 & - & - \\
\hline Être bien informée de la maîtrise du cancer & 29 & 85,3 & 4 & 11,8 & 1 & 2,9 & - & - \\
\hline $\begin{array}{l}\text { Être bien informée sur les avantages } \\
\text { du traitement }\end{array}$ & 30 & 88,2 & 2 & 5,9 & 2 & 5,9 & - & - \\
\hline Recevoir de l'information sur les rapports sexuels & 31 & 91,2 & 1 & 2,9 & 2 & 5,9 & - & - \\
\hline
\end{tabular}

Tableau 7. Items du domaine spirituel $(\mathrm{N}=34)$

\begin{tabular}{|c|c|c|c|c|c|c|c|c|}
\hline \multirow{3}{*}{ Item } & \multirow{2}{*}{\multicolumn{2}{|c|}{$\begin{array}{l}\text { Femmes sans } \\
\text { inquiétudes }\end{array}$}} & \multicolumn{6}{|c|}{ Femmes ayant des inquiétudes } \\
\hline & & & \multicolumn{2}{|c|}{ Détresse minime } & \multicolumn{2}{|c|}{ Détresse modérée } & \multicolumn{2}{|c|}{ Détresse élevée } \\
\hline & Nombre & $\%$ & Nombre & $\%$ & Nombre & $\%$ & Nombre & $\%$ \\
\hline Incertitude à propos de l'avenir & 15 & 44,1 & 9 & 26,5 & 6 & 17,6 & 4 & 11,8 \\
\hline Sentiments relatifs à la mort et au mourir & 20 & 58,8 & 5 & 14,7 & 1 & 17,6 & 3 & 8,8 \\
\hline Conserver une conception positive de la vie & 25 & 73,5 & 4 & 11,8 & 4 & 11,8 & 1 & 2,9 \\
\hline Désarroi « Pourquoi cela m’arrive-t-il à moi? » & 25 & 73,5 & 2 & 5,9 & 4 & 11,8 & 3 & 8,8 \\
\hline Trouver une signification à cette expérience & 27 & 79,4 & 6 & 17,6 & - & - & 1 & 2,9 \\
\hline Bien utiliser le temps dont on dispose & 28 & 82,4 & 1 & 2,9 & 3 & 8,8 & 2 & 5,9 \\
\hline $\begin{array}{l}\text { Le personnel soignant communique un } \\
\text { sentiment d'espoir }\end{array}$ & 29 & 85,3 & 2 & 5,9 & 2 & 5,9 & 1 & 2,9 \\
\hline
\end{tabular}


patients atteints de cancer du poumon (Corner, Plant, A'Hern et Bailey, 1996). Quoique les travaux doivent être approfondis dans ce domaine, c'est l'application de ce savoir dans la pratique qui semble représenter un défi de taille. Son application adéquate éviterait aux patients de voir leurs besoins demeurer insatisfaits et d'avoir une qualité de vie moindre.

Outre les difficultés physiques, les problèmes psychologiques, sociaux et émotionnels constituaient les enjeux le plus souvent rapportés par les femmes, en ce qui concerne la période entourant la tenue de l'enquête. Certains de ces problèmes ont été mentionnés par d'autres auteurs (Bradley, Davis et Chow, 2005; Bruera et coll., 2001; Kennett et Payne, 2005; Solano et Higginson, 2006). Cependant, étant donné la composition des items de l'instrument, il était impossible de déterminer, dans cette étude, si les difficultés des patientes n'avaient pas du tout été dégagées par l'équipe de soins de santé primaires ou si, au contraire, elles avaient été dégagées et que le processus de prise en charge avait été lancé. Les études à venir pourraient inclure une question supplémentaire afin de clarifier ce point.

Un ensemble croissant de preuves démontre qu'une intervention psychosociale peut améliorer le bien-être et la survie chez les patients atteints de cancer (Chow, Tsao et Harth, 2004; Kennett et Payne, 2005). Il est donc critique que l'on focalise l'attention sur ce domaine. Les soins doivent être axés sur la personne considérée dans sa globalité plutôt que sur les soins des tumeurs et sur la gestion des symptômes. À tout le moins, une discussion avec les patientes pourrait déboucher sur une compréhension commune entre la patiente et la prestataire sur ce qui risque d'être le plus utile du point de vue de la patiente. Les prestataires de soins de santé doivent reconnaître l'existence de problèmes d'ordre psychologique, tels que les craintes entourant la l'incapacité ou la détérioration physique, tout autant que les problèmes d'ordre physique. Ils doivent instaurer une communication et un dialogue ouverts entre eux et les patientes afin

Tableau 8. Items du domaine pratique

\begin{tabular}{|c|c|c|c|c|c|c|c|c|}
\hline \multirow{3}{*}{ Item } & \multirow{2}{*}{\multicolumn{2}{|c|}{$\begin{array}{l}\text { Femmes sans } \\
\text { inquiétudes }\end{array}$}} & \multicolumn{6}{|c|}{ Femmes ayant des inquiétudes } \\
\hline & & & \multicolumn{2}{|c|}{ Détresse minime } & \multicolumn{2}{|c|}{ Détresse modérée } & \multicolumn{2}{|c|}{ Détresse élevée } \\
\hline & Nombre & $\%$ & Nombre & $\%$ & Nombre & $\%$ & Nombre & $\%$ \\
\hline $\begin{array}{l}\text { Changements au niveau des routines et du } \\
\text { style de vie habituel }\end{array}$ & 20 & 58,8 & 6 & 17,6 & 5 & 14,7 & 3 & 8,8 \\
\hline Longs délais avant les rendez-vous cliniques & 23 & 67,6 & 4 & 11,8 & 3 & 8,8 & 4 & 11,8 \\
\hline Inquiétudes relatives à la situation financière & 25 & 73,5 & 2 & 5,9 & 3 & 8,8 & 4 & 11,8 \\
\hline $\begin{array}{l}\text { Inquiétudes relatives aux allées et venues entre } \\
\text { le domicile et le centre }\end{array}$ & 26 & 76,5 & 2 & 5,9 & 3 & 8,8 & 3 & 8,8 \\
\hline $\begin{array}{l}\text { Avoir davantage de choix quant au centre que } \\
\text { vous devez fréquenter }\end{array}$ & 32 & 94,1 & - & - & 1 & 2,9 & 1 & 2,9 \\
\hline $\begin{array}{l}\text { Avoir davantage de choix quant au spécialiste } \\
\text { en cancérologie }\end{array}$ & 32 & 94,1 & 1 & 2,9 & 1 & 2,9 & - & - \\
\hline $\begin{array}{l}\text { Avoir des choix sur les moments où vous } \\
\text { devez vous présenter... }\end{array}$ & 32 & 94,1 & 1 & 2,9 & 1 & 2,9 & - & - \\
\hline
\end{tabular}

Tableau 9. Les items le plus fréquemment mentionnés et la détresse correspondante

\begin{tabular}{|l|l|l|}
\hline & $\begin{array}{l}\text { Femmes de l'échantillon pour qui cet } \\
\text { élément est source de difficulté (n=34) }\end{array}$ & $\begin{array}{l}\text { Parmi celles pour qui il constitue } \\
\text { une difficulté, } \% \text { qui ont une } \\
\text { détresse allant de modérée à élevée }\end{array}$ \\
\hline Peur que le cancer se propage (Psy.) & 28 & 57,1 \\
\hline Peur que le cancer revienne (Psy.) & 25 & 64,0 \\
\hline Manque d'énergie (Ph.) & 23 & 56,5 \\
\hline $\begin{array}{l}\text { Ne pas pouvoir faire les choses que vous pouviez } \\
\text { faire auparavant (Ph.) }\end{array}$ & 22 & 59,1 \\
\hline Incertitude à propos de l'avenir (Sp.) & 19 & 52,6 \\
\hline $\begin{array}{l}\text { Inquiétudes à propos du souci que se font } \\
\text { vos proches (Soc.) }\end{array}$ & 19 & 63,2 \\
\hline Se sentir déprimé (Ém.) & 18 & 55,6 \\
\hline Craintes relatives à la douleur (Psy.) & 18 & 55,6 \\
\hline Essoufflement (Ph.) & 17 & 41,2 \\
\hline Ne pas pouvoir faire les travaux ménagers (Ph.) & 17 & 82,4 \\
\hline Remarque : Ph. = physique; Soc. = social; Psy. = psychologique; Ém. = émotionnel; Sp. = spirituel
\end{tabular}


de pouvoir les diriger vers les ressources qui conviennent à leurs besoins. La pertinence des références dépendra de la mesure dans laquelle le problème est éprouvé et si le patient souhaite ou non recevoir de l'aide à son sujet à ce moment-là.

Il faut donc mettre en place les structures et les processus qui permettront de cerner aisément les difficultés auxquels les patients font face. L'instrument utilisé dans le cadre de la présente étude a fourni le type d'évaluation détaillée couvrant l'ensemble des besoins en matière de soins de soutien. L'utilisation d'un tel instrument normalisé facilite la détermination des difficultés avec lesquelles les patients se débattent. Les professionnels de la santé ne devraient pas supposer qu'un problème n'existe pas simplement parce que le patient ne leur en a pas parlé. De récents travaux ont permis d'apprendre que de nombreux patients n'abordent pas, avec les prestataires de soins, les problèmes auxquels ils sont confrontés malgré les inquiétudes qu'ils ressentent à leur sujet (Fitch, Ung, Winterhoff et Steele, 2002). Certes, ce type d'instrument de recherche détaillé peut s'avérer d'une application difficile dans un milieu clinique à l'horaire chargé. En revanche, on pourrait sélectionner les items le plus fréquemment mentionnés par les participantes afin d'élaborer une version abrégée de l'instrument qui servirait alors à des fins de dépistage. Il serait plus aisé d'administrer un tel instrument de dépistage ou de triage dans une salle d'attente et les résultats obtenus pourraient être partagés avec l'équipe clinique durant le rendez-vous afin de lancer une discussion sur le genre d'aide que la personne aimerait recevoir et de cerner l'intervention et/ou la ou les référence(s) pertinente(s).

L'une des lacunes des données obtenues dans le cadre de notre recherche est le manque de clarté entourant la proportion des femmes atteintes de cancer du poumon qui aimerait réellement obtenir de l'aide pour un problème qu'elles éprouvent actuellement. L'observation clinique semblerait indiquer que ce ne sont pas nécessairement toutes les patientes éprouvant actuellement une difficulté qui voudraient recevoir de l'aide à un moment particulier ou auprès du personnel du centre de cancérologie. Il se peut qu'elles reçoivent un soutien auprès d'un autre organisme de santé et qu'elles jugent que la difficulté est sur le point d'être résolue. Les futures recherches devraient incorporer des items ou des méthodes de collecte des données permettant de clarifier les souhaits de la personne en matière d'obtention d'aide. Cela formerait une assise importante de la planification et de l'attribution futures des ressources.

\section{Références}

Ashbury, F.D., Findley, H., Reynolds, B., \& McKerracher, K. (1998). A Canadian survey of cancer patients' experiences: Are their needs being met? Journal of Pain and Symptom Management, 16(5), 298-306.

Bailey, A.J., Parmar, M.K.B., \& Stephens, R.J. (1998). Patientreported short-term and long-term physical and psychologic symptoms. Journal of Clinical Oncology, 16(9), 3082-3093.

Bonevski, B., Sanson-Fisher, R., Girgis, A., Burton, L., Cook, P., Boyes, A., \& the Supportive Care Review Group. (2000). Evaluation of an instrument to assess the needs of patients with cancer. Cancer, 88(1), 217-25.

Bradley, N., Davis, L., \& Chow, E. (2005). Symptom distress in patients attending an outpatient palliative radiotherapy clinic. Journal of Pain and Symptom Management, 30(2), 123-131.

Brown, J., \& Radke, K. (1998). Nutritional assessment, intervention, and evaluation of weight loss in patients with non-small cell lung cancer. Oncology Nursing Forum, 25(3), 547-53.

Bruera, E., Michaud, M., Vigano, A., Neumann, C., Watanabee, S., \&
Il est troublant d'observer qu'une forte proportion des femmes atteintes de cancer du poumon a indiqué ressentir une détresse allant de modérée à élevée concernant les problèmes auxquels elles faisaient face actuellement. Les données soulignent la nécessité d'offrir des ressources et des interventions appropriées. Dans certaines régions, les centres pourraient se tourner vers certains employés comme une infirmière en pratique avancée, en vue de fournir une partie des soins exigés. Les résultats de cette étude mettent en relief la présence de difficultés et forment le fondement qui appuie la création d'initiatives en soins de soutien cernant les interventions et élaborant des protocoles fondés sur des données probantes. Par exemple, une recension approfondie de la littérature pourrait être effectuée en vue d'explorer les trois problèmes le plus fréquemment cités par les patientes de cette étude et de mettre au point des interventions locales pour ces problèmes qui seraient alors mis à l'épreuve dans le cadre d'un essai clinique randomisé afin de comparer les soins standard et la nouvelle intervention. Enfin, les patientes pourraient faire l'objet d'un suivi dans le cadre d'une étude longitudinale en vue de déterminer les interventions qui ont donné de bons résultats et le pourquoi.

\section{Conclusion}

Les femmes atteintes de cancer du poumon peuvent avoir un certain nombre de besoins insatisfaits pour lesquels elles nécessitent de l'aide. Il importe de dégager ces besoins le plus efficacement possible afin que les patientes puissent obtenir de l'aide auprès des ressources appropriées. Des besoins physiques tel que le manque d'énergie et la douleur sont éprouvés par la majorité de ces femmes, mais certaines d'entre elles éprouvent également des besoins émotionnels, psychologiques, sociaux, spirituels, pratiques et informationnels. Il est donc nécessaire d'élaborer des interventions afin d'aider les femmes atteintes de cancer du poumon à composer avec leurs besoins.

\section{Remerciement}

Le financement de cette étude a été assuré par l'Ontario Lung Association dans le cadre d'une subvention de recherche de l'Ontario Respiratory Care Society.

Hanson, J. (2001). Multidisciplinary symptom control clinic in a cancer centre: A retrospective study. Supportive Care in Cancer, 9, 162-168.

Chapple, A., Ziebland, S., \& MacPherson, A. (2004). Stigma, shame, and blame experienced by patients with lung cancer: A qualitative study. British Medical Journal, 328, 1470-1474.

Charles, K., Sellick, S.M., Montesanto, B., \& Mohide, E.A. (1996). Priorities for cancer survivors regarding psychosocial needs. Journal of Psychosocial Oncology, 14(2), 57-72.

Chow, E., Tsao, M., \& Harth, T. (2004). Does psychosocial intervention improve survival in cancer? A meta-analysis. Palliative Medicine, 18, 25-31.

Corner, J., Plant, H., A’Hern, R., \& Bailey, C. (1996). Nonpharmacological intervention for breathlessness in lung cancer. Palliative Medicine, 10(4), 299-305.

Edmonds, P., Karlsen, S., Khan, S., \& Addington-Hall, J. (2001). A comparison of the palliative care needs of patients dying from chronic respiratory diseases and lung cancer. Palliative Medicine, 15(4), 287-95. 
Erridge, S. (2003). Treatment and survival of British Columbia patients with lung cancer. Paper presentation at the 10th International Conference on Lung Cancer, Vancouver, August.

Fallen, H., \& Schmidt, M. (2004). Prognostic value of depressive coping and depressions in survival of lung cancer patients. Psycho-Oncology, 13(5), 359-363.

Fitch, M., Ung, Y., Winterhoff, M., \& Steele, R. (May, 2002). Identifying supportive care needs of lung cancer patients. Paper presented at the Canadian Association of Psychosocial Oncology Annual Research Conference, Halifax, Nova Scotia, Canada.

Given, C.W., Given, B.A., \& Stommel, M. (1994). The impact of age, treatment, and symptoms on the physical and mental health of cancer patients. Cancer, 74, 2128-38.

Groff, L. (2002). The gender lens: A new look at women's lungs. Update ORCS, 18(3), 1, 4-5.

Hollen, P.J., Gralla, R.J., \& Rittenberg, C.N. (2004). Quality of life as a clinical trial endpoint: Determining the appropriate interval for repeated assessments in patients with advanced lung cancer. Supportive Care in Cancer, 12(11), 767-773.

Hopwood, P., \& Stephens, R.J. (1995). Symptoms at presentation for treatment in patients with lung cancer: Implications for the evaluation of palliative treatment. British Journal of Cancer 71, 633-36.

Hopwood, P., \& Stephens, R.J. (2000). Depression in patients with lung cancer: Prevalence and risk factors derived from quality-oflife data. Journal of Clinical Oncology, 18(4), 893-903.

Institut national du cancer du Canada (INCC). (2006). Statistiques canadiennes sur le cancer 2006. Toronto, Canada : Auteur.

John, L.D. (2001). Quality of life in patients receiving radiation therapy for non-small cell lung cancer. Oncology Nursing Forum, 28(5), 807-813.

Kennett, C., \& Payne, M. (2005). Understanding why palliative care patients "like day care" and "getting out." Journal of Palliative Care, 21(4), 292-298.

Kiteley, C. et Fitch, M.I. (2006) Comprendre les symptômes éprouvés par les sujets atteints du cancer du poumon. Revue canadienne de soins infirmiers en oncologie, 16(1), 31-36.

Krishnasamy, M., Wilkie, E., \& Haviland, J. (2001). Lung cancer health care needs assessment: Patients' and informal carers' responses to a national mail questionnaire survey. Palliative Medicine, 15(3), 213-27.

Maliski, S., Sarna, L., Evangelista, L., \& Padilla, G. (2003). The aftermath of lung cancer: Balancing the good and bad. Cancer Nursing, 26(3), 237-244.

Moore, K.A., Mery, C.M., Jaklitsch, M.T., Estocain, A.P., Bueno, R., Swanson, S.J., et al. (2003). Menopausal effects on presentation, treatment, and survival of women with non-small cell lung cancer. Annals of Thoracic Surgery, 76(6), 1789-1795.
Morasso, G., Capelli, M., Viterbori, P., DiLeo, S., Alberisio, A., Costantini, M., et al. (1999). Psychological and symptom distress in terminal cancer patients with met and unmet needs. Journal of Pain and Symptom Management, 17(6), 402-9.

O'Driscoll, M., Corner, J., \& Bailey, C. (1999). The experience of breathlessness in lung cancer. European Journal of Cancer Care, 8(1), 37-43.

Radzikowska, E., Glaz, P., \& Roszkowski, K. (2002). Lung cancer in women: Age, smoking, histology, performance status, stage, initial treatment and survival. Population-based study of 20,561 cases. Annals of Oncology, 13(7), 1087-93.

Sarna, L., Brown, J.K., Cooley, M.E., Williams, R.D., Chernecky, C., Padilla, G., \& Danao, L.L. (2005). Quality of life and meaning of illness of women with lung cancer. Oncology Nursing Forum Online, 32(1), E9-19.

Sarna, L., Evangelista, L., Tashkin, D., Padilla, G., Holmes, C., Brecht, M., \& Grannis, F. (2004). Impact of respiratory symptoms and pulmonary function on quality of life of long-term survivors of non-small cell lung cancer. Chest, 125(2), 439-445.

Sarna, L., Lindsey, A.M., Dean, H., Brecht, M.L., \& McCorkle, R. (1994). Weight change and lung cancer: Relationships with symptom distress, functional status and smoking. Research in Nursing \& Health, 17(5), 371-9.

Siegfried, J.M. (2001). Women and lung cancer: Does oestrogen play a role? Lancet Oncology, 2(8), 506-513.

Solano, J., \& Higginson, I. (2006). A comparison of symptom prevalence in far advanced cancer, AIDS, heart disease, chronic obstructive pulmonary disease and renal disease. Journal of Pain and Symptom Management, 31(1), 58-68.

Trippoli, S., Varani, M., Lucioni, C., \& Messori, A. (2001). Quality of life and utility in patients with non-small cell lung cancer. Pharmacoeconomics, 19(8), 855-863.

Voogt, E., van der Heide, A., van Leeuwen, A.F., Visser, A.P. Cleiren, M.P., Passchier, J., et al. (2005). Positive and negative affect after diagnosis of advanced cancer. Psychooncology, 14(4), 262-273.

Whelan, T.J., Mohide, E.S., Willan, A.R., Arnold, A., Tew, A., Sellick, S., et al. (1997). The supportive care needs of newly diagnosed cancer patients attending a regional cancer centre. Cancer, 80(8), $1518-1524$

Zabora, J., Brintzenhofeszoc, K., Curbow, B., Hooker, C., \& Piantadosi, S. (2001). The prevalence of psychological distress by cancer site. Psychooncology, 10(1), 19-28. 